\title{
Dynamic Algorithms for the Massively Parallel Computation Model
}

\author{
Giuseppe F. Italiano \\ LUISS University, Rome, Italy \\ Vahab S. Mirrokni \\ Google Research, New York, USA
}

\author{
Silvio Lattanzi \\ Google Research, Zurich, Switzerland \\ Nikos Parotsidis* \\ University of Copenhagen, Denmark
}

\begin{abstract}
The Massive Parallel Computing (MPC) model gained popularity during the last decade and it is now seen as the standard model for processing large scale data. One significant shortcoming of the model is that it assumes to work on static datasets while, in practice, real world datasets evolve continuously. To overcome this issue, in this paper we initiate the study of dynamic algorithms in the MPC model. We first discuss the main requirements for a dynamic parallel model and we show how to adapt the classic MPC model to capture them. Then we analyze the connection between classic dynamic algorithms and dynamic algorithms in the MPC model. Finally, we provide new efficient dynamic MPC algorithms for a variety of fundamental graph problems, including connectivity, minimum spanning tree and matching.
\end{abstract}

\section{CCS CONCEPTS}

- Theory of computation $\rightarrow$ Dynamic graph algorithms; MapReduce algorithms; Distributed computing models.

ACM Reference Format:

Giuseppe F. Italiano, Silvio Lattanzi, Vahab S. Mirrokni, and Nikos Parotsidis. 2019. Dynamic Algorithms for the Massively Parallel Computation Model. In 31st ACM Symposium on Parallelism in Algorithms and Architectures (SPAA '19), June 22-24, 2019, Phoenix, AZ, USA. ACM, New York, NY, USA, 10 pages. https://doi.org/10.1145/3323165.3323202

\section{INTRODUCTION}

Modern applications often require performing computations on massive amounts of data. Traditional models of computation, such as the RAM model or even shared-memory parallel systems, are inadequate for such computations, as the input data do not fit into the available memory of today's systems. The restrictions imposed by the limited memory in the available architectures has led to new models of computation that are more suitable for processing massive amounts of data. A model that captures the modern needs of computation at a massive scale is the Massive Parallel Computing (MPC) model, that is captured by several known systems (such as

\footnotetext{
${ }^{*}$ The author is supported by Grant Number 16582, Basic Algorithms Research Copenhagen (BARC), from the VILLUM Foundation. Work partially done while the author was an intern at Google.

Permission to make digital or hard copies of part or all of this work for personal or classroom use is granted without fee provided that copies are not made or distributed for profit or commercial advantage and that copies bear this notice and the full citation on the first page. Copyrights for third-party components of this work must be honored. For all other uses, contact the owner/author(s).

SPAA '19, June 22-24, 2019, Phoenix, AZ, USA

(C) 2019 Copyright held by the owner/author(s)

ACM ISBN 978-1-4503-6184-2/19/06

https://doi.org/10.1145/3323165.3323202
}

MapReduce, Hadoop, or Spark). At a very high-level, a MPC system consists of a collection of machines that can communicate with each other through indirect communication channels. The computation proceeds in synchronous rounds, where at each round the machines receive messages from other machines, perform local computations, and finally send appropriate messages to other machines so that the next round can start. The crucial factors in the analysis of algorithms in the MPC model are the number of rounds and the amount of communication performed per round.

The MPC model is an abstraction of a widely-used framework in practice and has resulted in an increased interest by the scientific community. An additional factor that contributed to the interest in this model is that MPC exhibits unique characteristics that are not seen in different parallel and distributed architectures, such as its ability to perform expensive local computation in each machine at each round of the computation. Despite its resemblance to other parallel models, such as the PRAM model, the MPC model has different algorithmic power from the PRAM model [23].

The ability of the MPC model to process large amounts of data, however, comes with the cost of the use of large volumes of resources (processing time, memory, communication links) during the course of the computation. This need of resources strengthens the importance of efficient algorithms. Although the design of efficient algorithms for solving problems in the MPC model is of vital importance, applications often mandate the recomputation of the solution (to a given problem) after small modifications to the structure of the data. For instance, such applications include the dynamic structure of the Web where new pages appear or get deleted and new links get formed or removed, the evolving nature of social networks, road networks that undergo development and constructions, etc. In such scenarios, even the execution of very efficient algorithms after few modifications in the input data might be prohibitive due to their large processing time and resource requirements. Moreover, in many scenarios, small modifications in the input data often have a very small impact in the solution, compared to the solution in the input instance prior to the modifications. These considerations have been the driving force in the study of dynamic algorithms in the traditional sequential model of computation.

Dynamic algorithms maintain a solution to a given problem throughout a sequence of modifications to the input data, such as insertions or deletion of a single element in the maintained dataset. In particular, dynamic algorithms are able to adjust efficiently the maintained solution by typically performing very limited computation. Moreover, they often detect almost instantly that the maintained solution needs no modification to remain a valid solution to the updated input data. The update time of a dynamic algorithm in 
the sequential model is the time required to update the solution so that it is a valid solution to the current state of the input data. Dynamic algorithms have worst-case update time $u(N)$ if they spend at most $O(u(N))$ after every update, and $u(N)$ amortized update bound if they spend a total of $O(k \cdot u(N))$ time to process a sequence of $k$ updates. The extensive study of dynamic algorithms has led to results that achieve a polynomial, and often exponential, speed-up compared to the recomputation of a solution from scratch using static algorithms, for a great variety of problems. For instance, computing the connected components of a graph takes $O(m+n)$ time, where $n$ and $m$ are the number of vertices and edges of the graph, respectively, while the most efficient dynamic algorithms update the connected components after an edge update in $O(\log n)$ amortized time [20], or in sub-polynomial time in the worst-case [27]. Similarly, there exist algorithms that maintain a maximal matching in polylogarithmic time per update in the worst case [10], while recomputing from scratch requires $O(m+n)$ time.

So far, there has been very little progress on modelling dynamic parallel algorithms in modern distributed systems, despite their potential impact in modern applications, with respect to the speedup and reduced use of resources. There have been few dynamic algorithms that maintain the solution to a problem in the distributed setting. For instance, in [11], Censor-Hillel et al. present a dynamic algorithm for maintaining a Maximal Independent Set of a graph in the LOCAL model. Assadi et al. [7] improve the message complexity by adjusting their sequential dynamic algorithm to the LOCAL model. In [2], Ahn and Guha study problems that can be fixed locally (i.e., within a small neighborhood of some vertex) after some small modification that has a very limited impact on the existing solution. This line of work has been primarily concerned with minimizing the number of rounds and the communication complexity. Moreover, the algorithms designed for the LOCAL model do not necessarily take into account the restricted memory size in each machine.

In this paper, we present an adaptation of the MPC model, that we call DMPC, that serves as a basis for dynamic algorithms in the MPC model. First, we impose a strict restriction on the availability of memory per machine, which mandates the algorithms in this model to operate in any system that can store the input in the total memory. Second, we define a set of factors that determine the complexity of a DMPC algorithm. These factors consist of (i) the number of rounds per update that are executed by the algorithm, (ii) the number of machines that are active per round, and (iii) the total amount of communication per round, which refers to the sum of sizes of all messages sent at any round. A final requirement for our model is that DMPC algorithms should provide worst-case update time guarantees. This is crucial not only because of the shared nature of the resources, but also because it is imposed by many realworld applications, in which one needs to act fast upon an update in the data, such as detecting a new malicious behavior, or finding relevant information to display to a new activity (e.g., displaying ads, friend recommendations, or products that are relevant to a purchase).

Inspired by today's systems that share their resources between many different applications at any point in time, it is necessary to design algorithms that do not require dedicated systems to operate on, and that can be executed with limited amounts of resources, such as memory, processors, and communication channels. This necessity is further strengthened by the fact that typically dynamic algorithms are required to maintain a solution to a problem over long series of updates, which implies that the application is running for a long sequence of time. Our model imposes these properties through the predefined set of restriction. In particular, we focus on three main dimensions

Memory. Dynamic algorithms in our model are required to use a very limited amount of memory in each machine. Specifically, assuming that the input is of size $N$, each machine is allowed to use only $O(\sqrt{N})$ memory. Note that this limitation does not aim at ensuring that the machines are large enough to fit $O(\sqrt{N})$ bits (as a system with such weak machines would need many millions of machines to even store the data, given that even weak physical machines have several GB of memory). Rather, it aims at guaranteeing that the allocation of the machines of the model to physical machines is flexible in terms of memory, allowing the system to move machines of the model across different physical machines without affecting the execution of the algorithm. (Notice that the system can co-locate several machines of the model to a single physical machine.)

Resource utilization and number of machines. Our model promotes limited processing time in several ways. First, two factors of evaluation of an algorithm are the number of rounds that are required to process each update, and the number of machines that are active at each round of the update. Notice that machines that are not used by the execution of a dynamic algorithm can process other applications that co-exist in the same physical machines. Moreover, algorithms with worst-case update time are guaranteed to end the execution of a particular update in limited time, thus avoiding locking shared resources for large periods of time.

Communication Channels. In our model, one of the factors that contributes to the complexity of an algorithm is the amount of communication that occurs at each round during every update. Furthermore, the number of machines that are active per round also contributes to the complexity of an algorithm (namely, the number of machines receiving or transmitting messages). These two facts ensure that efficient algorithms in the DMPC model use limited communication.

Similarly to the sequential model, the goal of a dynamic algorithm in the DMPC model is to maintain a solution to a problem more efficiently than recomputing the solution from scratch with a static algorithm. Here, the main goal is to reduce the bounds in all three factors contributing to the complexity of an algorithm. However, algorithms reducing some of the factors, without increasing the others, may also be of interest.

We initiate the study of dynamic algorithms in the DMPC model by designing algorithms for basic graph-theoretic problems. In particular, we present fully-dynamic algorithms for maintaining a maximal matching, a 3/2-approximate matching, a $(2+\epsilon)$-approximate matching, the connected components of a graph, as well as a $(1+\epsilon)$ approximate Minimum Spanning Tree (MST) of a weighted graph.

Finally, we show that our model can exploit successfully the techniques that were developed for dynamic algorithms in the sequential model. In particular, we present a black-box reduction that 
transforms any sequential dynamic algorithm with $p(S)$ preprocessing time and $u(S)$ update time to an algorithm in the dynamic MPC model which performs the preprocessing step in $O(p(S))$ rounds, uses $O(1)$ machines and $O(1)$ total communication per round, and such that each update is performed in $O(u(S))$ number or rounds using $O(1)$ machines and $O(1)$ total communication per round. With this reduction, the characteristics (amortized vs. worst-time and randomized vs. deterministic) of the DMPC algorithm are the same as the sequential algorithm.

Related work in the classic MPC model. It was known from the PRAM model how to compute a $(1+\epsilon)$ approximate matching in $O(\log n)$ rounds [26]. Lattanzi et al. [25] introduced the so-called filtering technique which gives an algorithm for computing a 2approximate matching in $O(1 / c)$ rounds assuming that the memory per machine is $O\left(n^{1+c}\right)$, for any $c>0$. Under the same memory assumption, Ahn and Guha [2] showed an algorithm running in $O(1 /(c \epsilon))$ number of rounds for $(1+\epsilon)$ approximate matching. Both those algorithms run in $O(\log n)$ time when the memory in each machine is $\Theta(n)$, which matches the known bound from the PRAM model. It was only recently that Czumaj et al. [14] overcame the $O(\log n)$ barrier for computing an approximate matching. In particular, in [14] the authors presented a $(1+\epsilon)$-approximate matching in $O\left((\log \log n)^{2}\right)$ time with $\widetilde{O}(n)$ memory per machine. This bound has been improved to $O(\log \log n)$ rounds, under the assumption of slightly superlinear memory per machine $[6,16]$. Very recently, Ghaffari and Uitto [17] presented an algorithm that uses only sublinear memory and can compute a $(1+\epsilon)$-approximate matching in $\widetilde{O}(\sqrt{\log \Delta})$ rounds, where $\Delta$ is the maximum degree in the graph.

Another central problem in the MPC model is the computation of the connected components of a graph. This problem can be solved in $O(\log n)$ rounds [24, 27]. In particular, the algorithm in [24] runs in $O(\log \log n)$ rounds on certain types of random graphs. In the case where each machine contains $O\left(n^{1+c}\right)$ memory, it is known how to compute the connected components of a graph in a constant number of rounds [25]. Under a well-known conjecture [31], it is impossible to achieve $o(\log n)$ on general graphs if the space per machine is $O\left(n^{1-c}\right)$ and the total space in all machines is $O(m)$. Very recently Andoni et al. [4] presented a new algorithm that uses sublinear memory and runs in $\tilde{O}(\log D)$ parallel rounds, where $D$ is the diameter of the input graph.

Our results. Throughout the paper we denote by $G=(V, E)$ the input graph, and we use $n=|V|, m=|E|$, and $N=m+n$. All bounds refer to worst-case update bounds. Our algorithmic results are summarized in Table 1 . All of our algorithms use $O(N)$ memory across all machines, and hence make use of $O(\sqrt{N})$ machines.

Maximal matching. Our first algorithm maintains fully-dynamically a maximal matching in $O(1)$ rounds per update in the worst case, while the number of machines that are active per rounds is $O(1)$, and the total communication per round is $O(\sqrt{N})$. The general idea in this algorithm, inspired from [29], is to use vertex-partitioning across the machines and additionally to store at one machine the last $\sqrt{N}$ updates in a buffer, together with the changes that each of these updates generated. We call this summary of updates and the changes that they trigger the update-history. Every time that an update arrives (i.e., an edge insertion or an edge deletion), the update-history is sent to the endpoints that are involved in the update, and each endpoint adjusts its data structure based on the update-history (that is, it updates its knowledge of which vertices among its neighbors are free), and further sends back (to the machine that maintains the update-history) any possible changes that the update might have triggered. The machines that maintain the endpoints of the updated edge might further communicate with one of their neighbors to get matched with them. Additional challenges arise from the fact that the neighborhood of a single vertex might not fit in a single machine.

For comparison, the best static MPC algorithm to compute a maximal matching in the static case runs in $O(\log \log n)$ when the space per machine is $\widetilde{O}(n)$ [16], $O(\sqrt{\log n})$ when the space is sublinear [17] and in $O(c / \delta)$ rounds when $N \in \Omega\left(n^{1+c}\right)$ and the space per machine is $\Omega\left(n^{1+\delta}\right)$ [25]. These algorithms use all the machines at each round and generate $\Omega(N)$ communication per round.

We note that although our algorithm has communication complexity $O(\sqrt{N})$ per round in the case where the available memory per machine is $O(\sqrt{N})$, the communication complexity is actually proportional to the number of machines used by the system. Namely, if we allow larger memory per machine then the communication complexity reduces significantly. Hence, in real-world systems we expect our algorithm to use limited communication per MPC round.

3/2-approximate matching. We further study the problem of maintaining a maximum cardinality matching beyond the factor 2 approximation given by a maximal matching. We present an algorithm for maintaining a 3/2-approximate matching that runs in $O(1)$ rounds, uses $O(\sqrt{N})$ machines and $O(\sqrt{N})$ communication per round. The best known static algorithm for computing a $O(1+\epsilon)$ approximate matching runs in $O(\log \log n)$ rounds in the case where the memory available in each machine is $\widetilde{O}(n)[6,14,16]$ or in $O(\sqrt{\log \Delta})$ rounds when the memory available in each machine is sublinear [31], where $\Delta$ the maximum degree in the graph.

$(2+\epsilon)$-approximate matching. Our algorithm for maintaining a maximal matching requires polynomial communication among the machines and the use of a coordinator machine. To overcome those restrictions, we explore the setting where we are allowed to maintain an almost maximal matching instead of a proper maximal matching. In other terms, at most an $\epsilon$ fraction of the edges of a maximal matching may be missing. In this setting, we show that we can adapt the fully-dynamic centralized algorithm by Charikar and Solomon [12] that has polylogarithmic worst-case update time. We note that our black-box reduction to the DMPC model yields a fully-dynamic algorithm with a polylogarithmic number of rounds. However we show how we can adapt the algorithm to run in $O(1)$ rounds per edge insertion or deletion, using $O(p o l y \log (n))$ number of active machines and total communication per round. ${ }^{1}$

Connected components and $(1+\epsilon)$ MST. We consider the problem of maintaining the connected components of a graph and the problem of maintaining a $O(1+\epsilon)$-approximate Minimum Spanning

\footnotetext{
${ }^{1}$ We note that one could adopt the algorithm from [10] to maintain a (proper) maximal matching with the same asymptotic bounds; however, that algorithm does not maintain a consistent matching throughout its execution, meaning that the maintained matching could be completely different between consecutive update operations, which is not a desirable property for many applications.
} 
Table 1: Algorithmic results achieved in this paper. The bounds presented in the first part of the table hold in the worst-case.

\begin{tabular}{l|ccc|c}
\multicolumn{1}{c|}{ Problem } & \#rounds & $\begin{array}{c}\text { \#active } \\
\text { machines }\end{array}$ & $\begin{array}{c}\text { Commun. } \\
\text { per round }\end{array}$ & Comments \\
\hline Maximal matching & $O(1)$ & $O(1)$ & $O(\sqrt{N})$ & $\begin{array}{c}\text { Use of a coordinator, } \\
\text { starts from an arbitrary graph. }\end{array}$ \\
\hline 3/2-app. matching & $O(1)$ & $O(n / \sqrt{N})$ & $O(\sqrt{N})$ & Use of a coordinator. \\
\hline$(2+\epsilon)$-app. matching & $O(1)$ & $\widetilde{O}(1)$ & $\widetilde{O}(1)$ & $\begin{array}{c}\text { Use of Euler tours, } \\
\text { Connected comps }\end{array}$ \\
\hline & $O(1)$ & $O(\sqrt{N})$ & $O(\sqrt{N})$ & starts from an arbitrary graph. \\
\hline$(1+\epsilon)$-MST & $O(1)$ & $O(\sqrt{N})$ & $O(\sqrt{N})$ & $\begin{array}{c}\text { The approx. factor comes } \\
\text { from the preprocessing, } \\
\text { starts from an arbitrary graph. }\end{array}$ \\
\hline
\end{tabular}

\begin{tabular}{c|ccc|c}
\hline \multicolumn{4}{c|}{ Results from reduction to the centralized dynamic model } \\
\hline Maximal matching & $O(1)$ & $O(1)$ & $O(1)$ & Amortized, randomized. \\
\hline Connected comps & $\widetilde{O}(1)$ & $O(1)$ & $O(1)$ & Amortized, deterministic. \\
\hline MST & $\widetilde{O}(1)$ & $O(1)$ & $O(1)$ & Amortized, deterministic.
\end{tabular}

Tree (MST) on a weighted graph. For both problems we present fully-dynamic deterministic algorithms that run in $O(1)$ rounds per update in the worst case, with $O(\sqrt{N})$ active machines and $O(\sqrt{N})$ total communication per round. Notice that, in order to maintain the connected components of a graph, it suffices to maintain a spanning forest of the graph. As it is the case also for centralized algorithms, the hard case is to handle the deletion of edges from the maintained spanning forest. The main ingredient in our approach is the use of Euler tour of a spanning tree in each connected component. This enables us to distinguish between different trees of the spanning forest, based on the tour numbers assigned to each of vertices of the trees, which we use to determine whether a vertex has an edge to particular part of a tree. Notice that to achieve such a bound, each vertex needs to known the appearance numbers of its neighbors in the Euler tour, which one cannot afford to request at each round as this would lead to $O(N)$ communication. We show how to leverage the properties of the Euler tour in order to avoid this expensive step. In the static case, the best known algorithm to compute the connected components and the MST of a graph requires $O(c / \delta)$ rounds when $N \in \Omega\left(n^{1+c}\right)$ and $S \in \Omega\left(n^{1+\delta}\right)$ [25]. In the case where $S \in o(n)$, [13] presented an algorithm to compute the connected components of a graph in $O(\log n)$ rounds, with all the machines and $\Omega(N)$ communication per round.

Bounds from the dynamic algorithms literature. We present a reduction to dynamic algorithms in the centralized computational model. More specifically, we show that if there exists a centralized algorithm with update time $u(m, n)$ and preprocessing time $p(m, n)$ on a graph with $m$ edges and $n$ vertices, then there exists a dynamic MPC algorithm which updates the solution in $O(u(m, n))$ rounds with $O(1)$ active machines per round and $O(1)$ total communication, after $p(m, n)$ rounds of preprocessing. The characteristics of the centralized algorithm (e.g., amortized or worst-case update time, randomized or deterministic) carry over to the MPC model.
This reduction, for instance, implies an amortized $\widetilde{O}(1)$ round fullydynamic DMPC algorithm for maintaining the connected components or the maximum spanning tree (MST) of a graph [20], and an amortized $O(1)$ round fully-dynamic DMPC algorithm for the maximal matching problem [30]. These algorithms however do not guarantee worst-case update time, which is important in applications. Moreover, the connected components and MST algorithms have super-constant round complexity.

Road map. In section 2 we introduce the model. Then in Section 3 we present our maximal matching algorithm, in Section 4 we present our 3/2-approximation. Our connected component algorithm, and the $(1+\epsilon)$-MST, are presented in Section 5. Finally, our $(2+\epsilon)$ approximation algorithm and the reduction are omitted due to lack of space, and are available in the full version of the paper.

\section{THE MODEL}

In this work we build on the model that was introduced by Karloff, Suri, and Vassilvitski [23], and further refined in [3, 9, 18]. This model is commonly referred to as the Massive Parallel Computing (MPC) model. In its abstraction, the MPC model is the following. The parallel system is composed by a set of $\mu$ machines $M_{1}, \ldots, M_{\mu}$, each equipped with a memory that fits up to $S$ bits. The machines exchange messages in synchronous rounds, and each machine can send and receive messages of total size up to $S$ at each round. The input, of size $N$, is stored across the different machines in an arbitrary way. We assume that $S, \mu \in O\left(N^{1-\epsilon}\right)$, for a sufficiently small $\epsilon$. The computation proceeds in rounds. In each round, each machine receives messages from the previous round. Next, the machine processes the data stored in its memory without communicating with other machines. Finally, each machines sends messages to other machines. At the end of the computation, the output is stored across the different machines and it is outputted collectively. The data output by each machine has to fit in its local memory and, hence, each machine can output at most $S$ bits. 
Since at each round all machines can send and receive messages of total size $S$, the total communication per round is bounded by $S \cdot \mu \in O\left(N^{2-2 \epsilon}\right)$. See [23] for a discussion and justification. When designing MPC algorithms, there are three parameters that need to be bounded:

- Machine Memory: In each round the total memory used by each machine is $O\left(N^{(1-\epsilon)}\right)$ bits.

- Total Memory: The total amount of data communicated at any round is $O\left(N^{(2-2 \epsilon)}\right)$ bits.

- Rounds: The number of rounds is $O\left(\log ^{i} n\right)$, for a small $i \geq 0$.

Several problems are known to admit a constant-round algorithm, such as sorting and searching [18].

Dynamic algorithms. In the centralized model of computation, dynamic algorithms have been extensively studied in the past few decades. The goal of a dynamic algorithm is to maintain the solution to a problem while the input undergoes updates. The objective is to update the solution to the latest version of the input, while minimizing the time spent for each update on the input. A secondary optimization quantity is the total space required throughout the whole sequence of updates.

A dynamic graph algorithm is called incremental if it allows edge insertions only, decremental if it allows edge deletions only, and fully-dynamic if it allows an intermixed sequence of both edge insertions and edge deletions. Most basic problems have been studied in the dynamic centralized model, and they admit efficient update times. Some of these problems include, connectivity and minimum spanning tree [20,28], approximate matching [5, 8, 10, 12, 29, 30], shortest paths $[1,15]$.

Dynamic algorithms in the DMPC model. Let $G=(V, E)$ be a graph with $n=|V|$ vertices and $m=|E|$ edges. In the general setting of the MPC model, where the memory of each machine is strictly sublinear in $n$, no algorithms with constant number of rounds are known even for very basic graph problems, such as maximal matching, approximate weighted matching, connected components. Recomputing the solution for each of those problems requires $O(\log n)$ rounds, the amount of data that is shuffled between any two rounds can be as large as $O(N)$, all the machines are active in each round, and all machines need to communicate with each other. Therefore, it is natural to ask whether we can update the solution to these problems after a small change to the input graph, using a smaller number of rounds, less active machines per round, and less total communication per round. Notice that bounding the number of machines that communicate immediately implies the same bound on the active machines per round. For convenience, we call active the machines that are involved in communication. The number of active machines also implies a bound on the amount of data that are sent in one round, as each machine has information at most equal to its memory (i.e., $O(\sqrt{N})$ bits). The complexity of a dynamic algorithm in the DMPC model can be characterized by the following three factors:

- The number of rounds required to update the solution.

- The number of machines that are active per round.

- The total amount of communication per round.

An ideal algorithm in the DMPC model processes each update using a constant number of rounds, using constant number of machines and constant amount of total communication. While such an algorithm might not always be feasible, a dynamic algorithm should use polynomially (or even exponentially) less resources than it's static counterpart in the MPC model.

Use of a coordinator. Distributed systems often host multiple jobs simultaneously, which causes different jobs to compete for resources. Additionally, systems relying on many machines to work simultaneously are prone to failures of either machines or channels of communication between the machines. Our model, allows solutions where all updates are sent to a single (arbitrary, but fixed) machine that keeps additional information on the status of the maintained solution, and then coordinates the rest of the machines to perform the update, by sending them large messages containing the additional information that it stores. Examples of such an algorithm is our algorithm for the maximal matching, and the $3 / 2$ approximate matching. In practice, the use of a coordinator might create bottlenecks in the total running time, since it involves transmission of large messages, and also makes the system vulnerable to failures (i.e., if the coordinator fails, one might not be able to recover the solution).

We note that the role of the coordinator in our matching algorithms is not to simulate centralized algorithms (as we do in our reduction from DMPC algorithms to dynamic centralized algorithms), i.e., to perform all computation at the coordinator machine while treating the rest of the machines as memory. In particular, we treat the coordinator as a buffer of updates and changes of the solution, and we communicate this buffer to the rest of the machines on a need-to-know basis.

Algorithmic challenges. The main algorithmic challenges imposed by our model are the sublinear memory (most of the algorithm known in the MPC model use memory in $\Omega(n))$ and the restriction on the number of machines used in every round. This second point is the main difference between the MPC and DMPC model and poses a set of new interesting challenges.

\section{FULLY-DYNAMIC DMPC ALGORITHM FOR MAXIMAL MATCHING}

In this section we present a deterministic fully-dynamic algorithm for maintaining a maximal matching with a constant number of rounds per update and a constant worst-case number of active machines per update, when the memory of each machine is $\Omega(\sqrt{N})$ bits, where $N=(m+n)$ and $m$ is the maximum number of edges throughout the update sequence. The communication per round is $O(\sqrt{N})$. Recall that our model introduces additional restrictions in the design of efficient algorithms. Specifically, the memory of each machine might not even be sufficient to store the neighborhood of a single vertex, which implies that the edges incident to a single vertex may be stored in polynomially many machines. In this framework, a scan of the neighbors of a single vertex requires a polynomially number of active machines in each round.

Our algorithm borrows an observation from the fully-dynamic algorithm for maximal matching of Neiman and Solomon [29], which has $O(\sqrt{m})$ worst-case update time and $O\left(n^{2}\right)$ space, or the same amortized update bound with $O(m)$ space. Specifically, Neiman and Solomon [29] observe that a vertex either has a low degree, or has 
only few neighbors with high degree. This allows us to treat vertices with large degree separately from those with relatively small degree. We call a vertex heavy if it has a large degree and light if it has a small degree. The threshold in the number of vertices that distinguishes light from heavy vertices is set to be $2 \sqrt{m}$. As the memory of each machine is $\Omega(\sqrt{m})$, we can fit the light vertices together with their edges on a single machine, but for heavy vertices we can keep only up to $O(\sqrt{m})$ of their edges in a single machine. Given that each vertex knows whether it is an endpoint of a matched edge, the only non-trivial update to be handled is when an edge $e=(x, y)$ of the matching is deleted and we have to check whether there exists an edge adjacent to $x$ or $y$ that can be added to the matching. Notice that if the neighborhood of each vertex fits in a single machine, then it is trivial to bound the number of rounds to update the solution, as it is sufficient to search for free neighbors of $x$ and $y$ that can be matched to those vertices. Such a search can be done in a couple of rounds by sending a message from $x$ and $y$ to their neighbors to ask whether they are free to join or not. However, this does not immediately bound the number of active machines per round.

Overview. Our algorithm keeps for each light vertex all the edges of its adjacency list in a single machine. For every heavy node we keep only $\sqrt{2 m}$ edges that we call alive. We call suspended the rest of the edges of $v$. We initially invoke an existing algorithm to compute a maximal matching in $O(\log n)$ rounds. Our algorithm always maintains a matching with the following invariant:

INVARIANT 3.1. No heavy vertex gets unmatched throughout the execution of the algorithm ${ }^{2}$.

If a new edge gets inserted to the graph, we simply check if we can add it to the matching (i.e., if both its endpoints are free). Now assume that an edge $(x, y)$ from the matching gets deleted. If both the endpoints are light, then we just scan their adjacency lists (that lie in a single machine) to find a replacement edge for each endpoint of $(x, y)$. If $x$ is heavy, then we search the $\sqrt{2 m}$ alive edges of $x$ and if we find a neighbor that is free we match it. If we cannot find a free neighbor of $x$, then among the (matched) $\sqrt{2 m}$ alive neighbors of $x$ there should exist a neighbor $w$ with a light mate $z$ (as otherwise the sum of degrees of the mates of neighbors of $x$ would exceed $m$ ), in which case we remove $(w, z)$ from the matching, we add $(x, w)$ to the matching, and we search the neighbors of the (light) vertex $z$ for a free neighbor to match $z$. If $y$ is heavy, we proceed analogously.

We build the necessary machinery in order to keep updated the aforementioned allocation of the adjacency lists to the available machines. This involves moving edges between machines whenever this is necessary, which introduces several challenges, since we cannot maintain updated the information in all machines with only $O(1)$ message exchange. On the other hand, we cannot allocate edges to an arbitrary number of machines. We deal with these issues by periodically updating the machines by taking advantage of the fact that we can send large messages from the coordinator.

Initialization and bookkeeping. Our algorithm makes use of $O(\sqrt{N})$ machines. We assume that all vertices of the graph contain

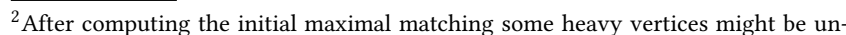
matched. During the update sequence, once a heavy vertex gets matched, it is not being removed from the matching, unless it becomes light again
}

IDs from 1 to $n$. Our algorithm executes the following preprocessing. First, we compute a maximal matching (this can be done in $O(\log n)$ rounds with the randomized CONGEST algorithm from [22]). Together with each edge in the graph we store whether an endpoint of the edge is matched: if it is, we also store its mates in the matching. In a second phase, we compute the degree of each vertex (this can be done in $O(1)$ rounds for all vertices). We place the vertices into the machines in such a way that the whole adjacency list of light vertices, and arbitrary $\sqrt{2 m}$ edges from the adjacency list of heavy vertices, are stored in single machines. The remaining adjacency list of a heavy vertex is stored in separate exclusive machines (only store edges of that vertex) so that as few machines as possible are used to store the adjacency list of a heavy vertex. On the other hand, the light vertices are grouped together into machines. The machines that store heavy vertices are characterized as heavy machines, and those storing light vertices as light machines.

One of the machines acts as the coordinator, in the sense that all the queries and updates are executed through it. The coordinator machine, denoted by $M_{C}$, stores an update-history $\mathcal{H}$ of the last $O(\sqrt{N})$ updates in both the input and the maintained solution, i.e., which edges have been inserted and deleted from the input in the last $\sqrt{N}$ updates and which edges have been inserted and deleted in the maintained maximal matching. Moreover, for each newly inserted edge that exists in the update-history we store a binary value for each of its endpoints that indicates if their adjacency list has been updated to include the edge.

For convenience, throughout this section we say that the algorithm invokes some function without specifying that all the communication is made through $M_{C}$. We dedicate $O(n / \sqrt{N})$ machines to store statistics about the vertices of the graphs, such as their degree, whether they are matched and who is their mate, the machine storing their alive edges, the last in the sequence of machines storing their suspended edges (we treat the machines storing suspended edges as a stack). To keep track of which machine keeps information about which vertices, we allocate many vertices with consecutive IDs to a single machine so that we can store the range of IDs stored in each machine. Hence in $M_{C}$, except of the updatehistory $\mathcal{H}$, we also store for each range of vertex IDs the machine that contains their statistics. This information fits in the memory of $M_{C}$ as the number of machines is $O(\sqrt{N})$. Finally, $M_{C}$ also stores the memory available in each machine.

Maintaining the bookkeeping. In what follows, for the sake of simplicity, we assume that the update-history $\mathcal{H}$ is updated automatically. Further, we skip the description of the trivial update or queries on the statistics of a vertex, such as its degree, whether it is an endpoint of a matched edge, the machine storing its alive edges, etc. All of these can be done in $O(1)$ rounds via a message through the coordinator machine $M_{C}$. After each update to the graph, we update the information that is stored in a machine by executing those updates in a round-robin fashion, that is, each machine is updated after at most $O(\sqrt{N})$ updates. Recall that we use $O(\sqrt{N})$ machines.

Throughout the sequence of updates we use the following set of supporting procedures to maintain a valid allocation of the vertices into machines: 
- getAlive $(x)$ : Returns the ID of the machine storing the alive neighbors of $x$.

- getDegInMachine $(M, x)$ : Returns $x$ 's degree in machine $M$.

- getSuspended $(x):$ Returns the ID of the last in the sequence of heavy machines storing the edges of $x$.

- $f$ its $(M, s)$ : Return true if $s$ edges fit into a light machine $M$, and false otherwise.

- toFit(s) : Returns the ID of a light machine that has enough memory to store $s$ edges, and the available space in that machine.

- addEdge $((x, y))$ : We only describe the procedure for $x$, as the case for $y$ is completely analogous. If $x$ is heavy, add $(x, y)$ in the machine getSuspended $(x)$ if it fits, or otherwise to a new machine, and set the new machine to be getSuspended( $x)$. If, on the other hand, $x$ is light and $(x, y)$ fits into getAlive $(x)$, we simply add $(x, y)$ in $\operatorname{get} \operatorname{Alive}(x)$. If, $(x, y)$ does not fit in getAlive $(x)$ then call moveEdges $\left(x, s, M_{x}, \operatorname{toFit}(s), \mathcal{H}\right)$, where $s$ is the number of alive edges of $x$ (if $x$ becomes heavy, we mark that). If all of the remaining edges in the machine $M_{x}$ (of light vertices other than $x$ ) fit into another machine, then move them there (this is to bound the number of used machines).

- moveEdges $\left(x, s, M_{1}, M_{2}, \mathcal{H}\right)$, where $x$ is light: First, remove from machine $M_{1}$ deleted edges of $x$ based on $\mathcal{H}$. Second, send from $M_{1}$ up to $s$ edges of $x$ to $M_{2}$. If the $s$ edges do not fit into $M_{2}$, move the neighbors of $x$ from $M_{2}$ to a machine that fits them, i.e., execute $M_{\mathcal{X}^{\prime}}=\operatorname{toFit}(s+$ getDegInMachine $(M, x))$, move the $s$ edges of $x$ in $M_{1}$ to $M_{x^{\prime}}$ and call moveEdges $\left(x, \operatorname{getDegInMachine}(M, x), M_{2}, M_{x^{\prime}}, \mathcal{H}\right)$.

- fetchSuspended $(x, s)$, where $x$ is heavy: Moves $s$ suspended edges to the machine $M_{x}=$ getAlive $(x)$. To achieve this we call moveEdges $\left(x, s\right.$, getSuspended $\left.(x), M_{x}\right)$. While the number of edges moved to $M_{x}$ is $s^{\prime}<s$, call moveEdges $\left(x, s-s^{\prime}\right.$,getSuspended $\left.(x), M_{x}\right)$.

$-\operatorname{moveSuspended}(x, s, L)$, where $x$ is heavy: Moves the set $L$ of $s$ edges of $x$ from machine getAlive $(x)$ to the machines storing the suspended edges of $x$. We first fit as many edges as possible in the machine getSuspended $(x)$, and the rest (if any) to a new machine.

- updateVertex $(x, \mathcal{H})$ : Update the neighbors of $x$ that are stored in $M_{x}=\operatorname{get} \operatorname{Alive}(x)$ based on $\mathcal{H}$. If $x$ is heavy and the number of edges from the adjacency list of $x$ in $M$ is $s<\sqrt{2 m}$, then call fetchSuspended $(x, \sqrt{2 m}-s)$. If $x$ is heavy and the set of alive edges has size $s>\sqrt{2 m}$, then call moveSuspended $(x, s-\sqrt{2 m}, L)$, where $L$ are $s-\sqrt{2 m}$ edges of $x$ that do not contain the edge $(x$, mate $(x))$. If, on the other hand, $x$ is light and the set of alive edges of $x$ does not fit in $M_{x}$ after the update, call moveEdges $\left(x, s, M_{x}\right.$, toFit $\left.(s), \mathcal{H}\right)$, where $s$ is the number of alive edges of $x$. If all of the remaining edges in the machine $M_{x}$ (of light vertices other than $x$ ) fit into another machine, then move them there (this is to bound the number of used machines).

- updateMachine $(M, \mathcal{H})$ : Update all adjacency lists stored in machine $M$ to reflect the changes in the update-history $\mathcal{H}$. If $M$ is a heavy machine of a vertex $x$, we proceed as in the case of updateVertex $(x, \mathcal{H})$, but now on machine $M$ rather than getAlive $(x)$. Now we assume $M$ is light. First, delete the necessary edges of the light vertices stored at $M$ based on $\mathcal{H}$. If all of the remaining edges of the machine fit into another half-full machine, then move them there (this is to bound the number of used machines).

Handling updates. Now we describe how our algorithm updates the maintained maximal matching after an edge update. insert $(x, y)$. First, execute updateVertex $(x)$, updateVertex $(y)$, and addEdge $((x, y))$. If both $x$ and $y$ are matched then do nothing and return. If neither $x$ nor $y$ are matched, add $(x, y)$ to the matching and return. In the case where $x$ is matched and heavy and $y$ is unmatched and light then do nothing and return. The same happens if $y$ is matched and heavy and $x$ is unmatched. If $x$ is unmatched and heavy, search for a (matched, as this is a maximal matching) neighbor $w$ of $x$ whose mate $z$ is light, remove $(w, z)$ from the matching, add $(x, w)$ to the matching, and if $z$ (who is a light vertex) has an unmatched neighbor $q$ add $(z, q)$ to the matching. If $y$ is unmatched and heavy proceed analogously. Note that this restores Invariant 3.1. In any case, the update-history is updated to reflect all the changes caused by the insertion of $(x, y)$.

delete $(x, y)$. First, update $\mathcal{H}$ to reflect the deletion of $(x, y)$ and call updateVertex $(x)$ and updateVertex $(y)$. If $(x, y)$ is not in the matching do nothing and return. (The edge has already been deleted from the adjacency lists via the calls to updateVertex.) If $(x, y)$ is in the matching proceed as follows. First, remove $(x, y)$ from the matching. If $z \in\{x, y\}$ is heavy, search for a neighbor $w$ of $z$ whose mate $w^{\prime}$ is light, remove $\left(w, w^{\prime}\right)$ from the matching, add $(z, w)$ to the matching, and if $w^{\prime}$ (who is a light vertex) has an unmatched neighbor $q$ add $\left(w^{\prime}, q\right)$ to the matching. If $z \in\{x, y\}$ is light, scan the neighborhoods of $z$ for a unmatched vertex $w$, and add $(z, w)$ to the matching. In any case, the update-history is updated to reflect all the changes caused by the deletion of $(x, y)$.

Lemma 3.2. The algorithm uses $O(\sqrt{N})$ machines.

Proof. Omitted due to lack of space.

Lemma 3.3. Both insert $(x, y)$ and delete $(x, y)$ run in $O(1)$ rounds, use $O(1)$ machines, and generate $O(\sqrt{N})$ communication per round.

Proof. Recall that we manage the machines that are used to store the sequence of machines storing the suspended edges of heavy vertices as stacks, that is, we store the last machine storing the suspended edges of a vertex $x$ together with the rest of the statistics for $x$, and each machine maintains a pointer to the next machine in the sequence. Hence, we can access in $O(1)$ rounds the machine that is last in the sequence of machines maintaining the suspended edges of a vertex. The only supporting function that is not trivially executable in $O(1)$ rounds is fetchSuspended. Note that a call to fetchSuspended makes multiple calls to moveEdges to transfer edges suspended edges of a heavy vertex $x$. As each machine is updated every $O(\sqrt{N})$ rounds, it follows that the number of edges that have been removed from the graph and the machines storing those edges are not yet updated, is $O(\sqrt{N})$. As all the calls to moveEdges transfer at most $O(\sqrt{N})$ edges of $x$, and all but one machines storing suspended edges of $x$ are full, it follows that there is at most a constant number of calls to moveEdges.

\section{FULLY-DYNAMIC 3/2-APPROXIMATE MAXIMUM MATCHING}

The algorithm for the $3 / 2$ approximate matching builds on top of the algorithm for maintaining a maximal matching from Section 3. Our algorithm is an adaptation of the algorithm from [29] to our DMPC model. Our algorithm's approximation is based on 
a well-known graph-theoretic connection between augmenting paths in an unweighted graph, with respect to a matching, and the approximation factor of the matching relatively to the maximum cardinality matching. An augmenting path is a simple path starting and ending at a free vertex, following alternating unmatched and matched edges. Specifically, a matching that does not contain augmenting paths of length $(2 k-1)$ in a graph, is a $\left(1+\frac{1}{k}\right)$-approximate matching [21]. In this section we show that it is possible to use the technique in [29] to design a simple DMPC algorithm for $k=2$. The additional information that the algorithm needs to maintain, compared to the algorithm from Section 3, is the number of unmatched neighbors of each vertex. We call these counters free-neighbor counters of the light vertices. We keep this information in the $O(n / \sqrt{N})$ machines storing the statistics about the vertices of the graph. Here assume that the computation starts from the empty graphs (An initialization algorithm for this problem would require eliminating all augmenting paths of length 3 , but we are not aware of such an algorithm using $O(N)$ total memory).

Since the algorithm from Section 3 maintains a matching where all heavy vertices are always matched, we only update the freeneighbor counters whenever a light vertex changes its matching status. Recall that a light vertex keeps all of its neighbors in the same machine. Therefore, we simply update the counters of the neighbors of the light vertex. This requires a message of size $O(\sqrt{N})$ from the light vertex $v$ that changed its status to the coordinator and from there appropriate messages of total size $O(\sqrt{N})$ to the $O(n / \sqrt{N})$ machines storing the free-neighbor counters of the neighbors of $v$.

Given that we maintain for each vertex its free-neighbor counter, we can quickly identify whether an edge update introduces augmenting paths of length 3 . The modifications to the algorithm from Section 3 are as follows.

- In the case of the insertion of edge $(u, v)$, if $u$ is matched but $v$ unmatched, we check whether the mate $u^{\prime}$ of $u$ has a free neighbor $w$; if so, we remove $\left(u, u^{\prime}\right)$ from the matching and we add $\left(w, u^{\prime}\right)$ and $(u, v)$ (this is an augmenting path of length 3$)$. We only update the free-neighbor counters of the neighbors of $w$ and $v$, as no other vertices change their status, and no new augmenting paths are introduces as no matched vertex gets unmatched.

- If $u$ and $v$ are free after inserting $(u, v)$, we add $(u, v)$ into the matching and update the free-neighbor counters of all neighbors of $u$ and $v$ (who are light vertices, as all heavy vertices are matched).

- If we delete an edge which is not in the matching, then we simply update the free-neighbor counters of its two endpoints.

- Whenever an edge $(u, v)$ of the matching is deleted, we treat $u$ as follows. If $u$ has a free neighbor $w$, then we add $(u, w)$ to the matching and update the free-neighbor counters of the neighbors of $w$ (who is light). If $u$ is light but has no free neighbors, then we search for an augmenting path of length 3 starting from $u$. To do so, it is sufficient to identify a neighbor $w$ of $u$ whose mate $w^{\prime}$ has a free neighbor $z \neq u$. If there exists such $w^{\prime}$ then we remove $\left(w, w^{\prime}\right)$ from the matching and add $(u, w)$ and $\left(w^{\prime}, z\right)$ to the matching, and finally update the free-neighbor counters of the neighbors of $z$ (who is light). No other vertex changes its status. If on the other hand, $u$ is heavy, then we find an alive neighbor $w$ of $u$ with a light mate $w^{\prime}$, remove $\left(w, w^{\prime}\right)$ from the matching and add $(u, w)$ to it. (This can be done in $O(1)$ rounds communication through the coordinator with the, up to $n / \sqrt{N}$, machines storing the mates of the statistics of the $O(\sqrt{N})$ alive neighbors of $w^{\prime}$.) Finally, given that $w^{\prime}$ is light, we proceed as before trying to either match $w^{\prime}$ or find an augmenting path of length 3 starting from $w^{\prime}$. Then, we proceed similarly to the case where $u$ was light.

Notice that in all cases where we have to update the free-neighbor counters of all neighbors of a vertex $v, v$ is a light vertex, so there are at most $O(\sqrt{N})$ counters to be updated and thus they can be accessed in $O(1)$ rounds, using $O(n / \sqrt{N})$ active machines, and $O(\sqrt{N})$ communication complexity. Hence, given the guarantees from Section 3 and the fact that we only take a constant number of actions per edge insertion or deletion, we conclude that our algorithm updates the maintained matching in $O(1)$ rounds, using $O(n / \sqrt{N})$ machines and $O(\sqrt{N})$ communication per round in the worst case. We conclude this section by proving the approximation factor of our algorithm.

LEMMA 4.1. The algorithm described in this section correctly maintains a 3/2-approximate matching.

Proof. Omitted due to lack of space.

\section{FULLY-DYNAMIC CONNECTED COMPONENTS AND APPROXIMATE MST}

In this section we present a fully-dynamic deterministic distributed algorithm for maintaining the connected components of a graph with constant number of rounds per edge insertion or deletion, in the worst case ${ }^{3}$. At the heart of our approach we use Euler tours, which have been successfully used in the design of dynamic connectivity algorithms in the centralized model of computation, e.g., in $[19,20]$. Given a rooted tree $T$ of an undirected graph $G$, an Euler tour (in short, E-tour) of $T$ is a path along $T$ that begins at the root and ends at the root, traversing each edge exactly twice. The E-tour is represented by the sequence of the endpoints of the traversed edges, that is, if the path uses the edges $(u, v),(v, w)$, then $v$ appears twice. As an E-tour is defined on a tree $T$, we refer to the tree $T$ of an E-tour as the Euler tree (E-tree, in short) of the E-tour. The root of the E-tree appears as the first and as the last vertex of its E-Tour. The length of a tour of an E-tree $T$ is ELength $h_{T}=4(|T|-1)$, the endpoints of each edge appear twice in the E-tour. See Figures 1 and 2 for examples. As the preprocessing shares similarities with the edge insertion, we postpone the description of the preprocessing after describing the update procedure to restore the E-tour after an edge insertion or deletion.

We assume that just before an edge update, we maintain for each connected component of the graph a spanning tree, and an E-tour of the spanning tree. Using vertex-based partitioning we distribute the edges across machines, and each vertex is aware of the ID of its component, and together with each of its edges we maintain the ID of the component that it belongs to and the two indexes in the E-tour (of the tree of the component) that are associated with the edge. Moreover, we maintain with each vertex $v$ the index of its first and last appearance in the E-tour of its E-tree, which we denote

\footnotetext{
${ }^{3}$ Note that no constant round algorithm for connected component is known for the static case. On the downside, the number of active machines per round is not bounded. We leave as an interesting area of future work to design an algorithm that uses a smaller number of machines per update
} 

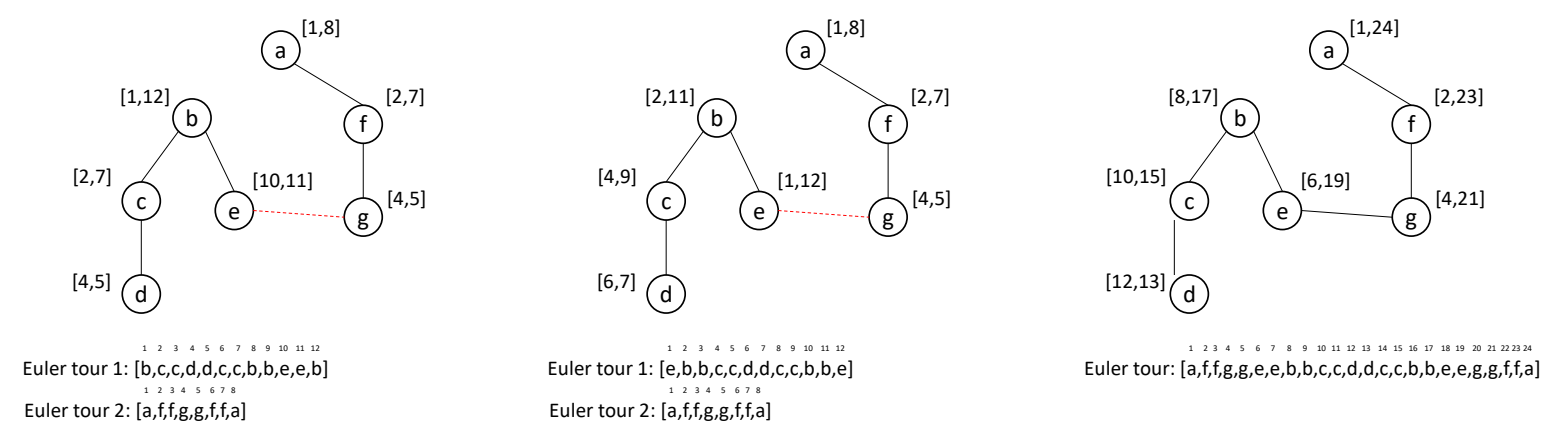

Figure 1: (i) A forest and an E-tour of each of its tree below. The brackets represent the first and the last appearance of a vertex in the E-tour. (ii) The E-tour after setting $e$ to be the root of its tree. (iii) The E-tour after the insertion of the edge $(e, g)$.
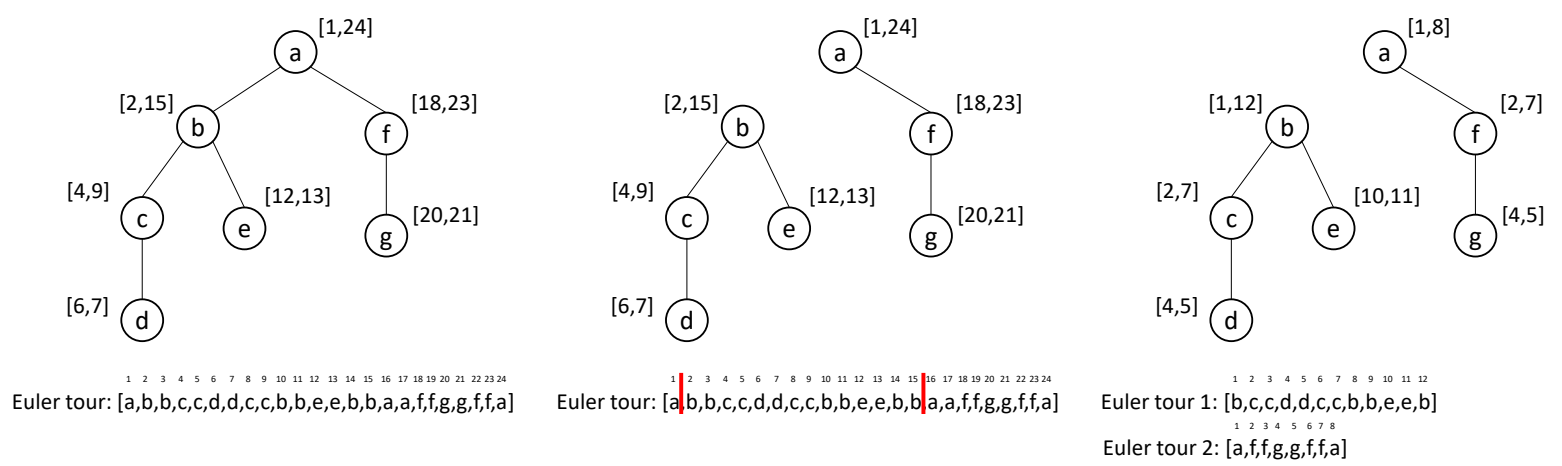

Figure 2: (i) A tree and an E-tour of the tree below it. The brackets represent the first and the last appearance of a vertex in the E-tour. (ii) An intermediate step of the update of the E-tour after the deletion of the edge $(a, b)$. The red lines in the E-tour indicate the split points of outdated E-tour. (iii) The E-tour after the deletion of the edge $(a, b)$.

by $f(v)$ and $l(v)$. We denote by index $v$ the set of all indexes that $v$ appears in the E-tour of $T$. Note that $\mid$ index $_{v} \mid=2 \cdot d_{T}(v)$ in the E-tour, where $d_{T}(v)$ is the degree of $v$ in the corresponding E-tree $T$. We do not explicitly store index $x_{v}$, this is implicitly stored with each vertex as information on $v$ 's edges. Therefore, we perform updates on the indexes in index $v$ but it is actually the indexes that are stored at the edges that are updated. To update this information in a distributed fashion, we leverage the properties of an E-tour which allows us to change the root of an E-tree, merge two E-trees, and split an E-tree, by simply communicating the first and last indexes of the new root, or the endpoints of the inserted/deleted edge.

Handling updates. The main idea to handle updates efficiently is that the E-tour of the spanning trees can be updated efficiently with limited communication. For instance, we change the root of an E-tree, and update all the information stored in the vertices of that tree, by sending $O(1)$-size messages to all vertices. Moreover, we test whether a vertex $u$ is an ancestor of a vertex $v$, in their common E-tree, using only the values $f(u)$ and $l(u)$ and $f(v)$ and $l(v)$. We handle the edge updates as follows.

insert $(x, y)$ : If $x$ and $y$ are in the same connected component, we simply add the edge to the graph. Otherwise, we proceed as follows. We first make $y$ the root of its E-tree $T_{y}$ (if it is not already). Let ELength $h_{T_{y}}=4\left(\left|T_{y}\right|-1\right)$ denote the length of the E-tour of $T_{y}$. For each vertex $w$ in $T_{y}$ we update each index $i \in$ index $_{w}$ to be $i=\left(\left(i+\right.\right.$ ELength $\left._{T_{y}}-l(y)\right) \bmod$ ELengt $\left._{T_{y}}\right)+1$. These shifts of the indexes of $w$ correspond to a new E-tour starting with the edge between $y$ and its parent, where the parent is defined with respect to the previous root of $T_{y}$. Second, we update the indexes $i \in$ index $_{w}$ of the vertices $w \in T_{y}$ to appear after the first appearance of $x$ in the new E-tour. For each vertex $w$ in $T_{y}$ update each index $i \in$ index $_{w}$ to be $i=i+f(x)+2$. Third, set index $y=$ index $_{y} \cup\{f(x)+2, f(x)+$ $l(y)+3\}$ and inde $_{x}=$ index $_{x} \cup\{f(x)+1, f(x)+l(y)+4\}$, where $l(y)$ is the largest index of $y$ in the E-tour of $T_{y}$ before the insertion of $(x, y)$. Finally, to update the indexes of the remaining vertices in $T_{x}$, for each $i \in$ index $_{w}$ where $i>f(x)$ we set $i=i+4 \cdot$ ELengt $_{T_{y}}$. See Figure 1 for an illustration.

Notice that the only information required by each vertex $w$ to perform this update, besides index $x_{w}$ which is implicitly stored on the edges of $w$ and $f(w)$, is ELength $T_{T_{y}}, f(y), l(y), f(x), l(x)$, which can be sent to all machines via a constant size message from $x$ and $y$ to all other machines. Notice that $x$ and $y$ do not need to store $f(x), l(x)$ and $f(y), l(y)$, ELengt $_{T_{y}}$, respectively, as they can simply learn those by sending and receiving an appropriate message to all machines. Hence each insertion can be executed in $O(1)$ rounds using all machines and $O(\sqrt{N})$ total communication per round (as all communication is between $x$ or $y$ with all other machines, and contains messages of $O(1)$ size).

delete $(x, y)$ : If $(x, y)$ is not a tree edge in the maintained forest, we simply remove the edge from the graph. Otherwise, we first split the E-tree containing $x$ and $y$ into two E-trees, and then we reconnect it if we find an alternative edge between the two E-trees. 
We do that as follows. We check whether $x$ is an ancestor of $y$ or vice versa by checking whether $f(x)<f(y)$ and $l(x)>l(y)$. Assume w.l.o.g. that $x$ is an ancestor of $y$ in $T_{x}$. First, we set index $x=$ inde $_{x} \backslash\{f(y)-1, l(y)+1\}$ and inde $x_{x}=$ inde $_{y} \backslash\{f(y), l(y)\}$ (that is, we simply drop the edge $(x, y))$. Then, for all descendants $w$ of $y$ in $T_{y}$ (including $y$ ), for each $i \in$ index $_{w}$ set $i=i-f(y)$, where $f(y)$ is the smallest index of $y$ before the deletion of $(x, y)$. Update $\left|T_{y}\right|$ and allocate a new ID for the new connected component containing $y$. Second, for all vertices $w \in T_{x} \backslash T_{y}$ and all $i \in$ index $_{w}$ if $i>l(y)$ set $i=i-((l(y)-f(y)+1)+2)$, where $l(y)$ and $f(y)$ are the largest and smallest, respectively, index of $y$ before the deletion of $(x, y)$. This is to inform all vertices that appear after $l(y)$ that the subtree rooted at $y$ has been removed, and hence the E-tour just cuts them off (the +2 term accounts for the two appearances of $\mathrm{x}$ in the E-tour because of $(x, y))$. Finally, we find an edge from a vertex $v \in T_{y}$ to a vertex $w \in T_{x}$, and execute $\operatorname{insert}(x, y)$.

Similarly to the case of an edge insertion, all of the above operations can be executed in a constant number of rounds as the only information that is required by the vertices are the ID of the components of $x$ and $y$, and the values $f(x), l(x), f(y), l(y)$, which are sent to all machines. Moreover, the search of a replacement edge to reconnect the two trees of $x$ and $y$ can be done in $O(1)$ rounds as we only need to send the IDs of the two components to all machines, and then each machine reports at most one edge between these two components to a specific machine (specified also in the initial message to all machines).

\subsection{Extending to $(1+\epsilon)$-approximate MST}

To maintain a minimum spanning tree instead of a spanning tree, we use the dynamic spanning tree algorithm with the following two changes. First, whenever an edge $(x, y)$ is added and the two endpoints are already in the same tree, we compute the edge $(u, v)$ with the maximum weight among all the edges whose both endpoints are ancestors of either $x$ or $y$ (but not both) and we compare it to the weight of $(x, y)$ (these tests can be done efficiently using the E-tree). We only keep the edge with the minimum weight among $(u, v)$ and $(x, y)$. Second, at Step 3 of the delete operation, instead of adding any edge between the two trees, the algorithm adds the minimum among all such edges.

The preprocessing can be adjusted to compute a $(1+\epsilon)$-approximate MST by doing bucketization, which introduces only a $O(\log n)$ factor in the number of rounds. In fact, it is enough to bucket the edges by weights and compute connected components by considering the edges in bucket of increasing weights iteratively and separately.

\section{REFERENCES}

[1] I. Abraham, S. Chechik, and S. Krinninger. Fully dynamic all-pairs shortest paths with worst-case update-time revisited. In Proc. of the 28th Annual ACM-SIAM Symp. on Discrete Algorithms, pages 440-452, 2017.

[2] K. J. Ahn and S. Guha. Access to data and number of iterations: Dual primal algorithms for maximum matching under resource constraints. ACM Transactions on Parallel Computing (TOPC), 4(4):17, 2018.

[3] A. Andoni, A. Nikolov, K. Onak, and G. Yaroslavtsev. Parallel algorithms for geometric graph problems. In Proc. of the 46th annual ACM Symp. on Theory of computing, pages 574-583. ACM, 2014.

[4] A. Andoni, Z. Song, C. Stein, Z. Wang, and P. Zhong. Parallel graph connectivity in $\log$ diameter rounds. In 59th IEEE Annual Symp. on Foundations of Computer Science, FOCS 2018, pages 674-685, 2018.

[5] M. Arar, S. Chechik, S. Cohen, C. Stein, and D. Wajc. Dynamic Matching: Reducing Integral Algorithms to Approximately-Maximal Fractional Algorithms. In 45th
International Colloquium on Automata, Languages, and Programming (ICALP 2018), volume 107, pages 7:1-7:16, 2018.

[6] S. Assadi, M. Bateni, A. Bernstein, V. Mirrokni, and C. Stein. Coresets meet edcs: algorithms for matching and vertex cover on massive graphs. In Proc. of the 30th Annual ACM-SIAM Symp. on Discrete Algorithms, pages 1616-1635, 2019.

[7] S. Assadi, K. Onak, B. Schieber, and S. Solomon. Fully dynamic maximal independent set with sublinear update time. In Proc. of the 50th Annual ACM SIGACT Symp. on Theory of Computing, STOC 2018, pages 815-826, 2018.

[8] S. Baswana, M. Gupta, and S. Sen. Fully dynamic maximal matching in $\mathrm{O}(\mathrm{log}$ n) update time. In IEEE 52nd Annual Symp. on Foundations of Computer Science, pages 383-392, 2011.

[9] P. Beame, P. Koutris, and D. Suciu. Communication steps for parallel query processing. In Proc. of the 32nd ACM SIGMOD-SIGACT-SIGAI Symp. on Principles of database systems, pages 273-284. ACM, 2013.

[10] A. Bernstein, S. Forster, and M. Henzinger. A deamortization approach for dynamic spanner and dynamic maximal matching. In Proc. of the 31st Annual ACM-SIAM Symp. on Discrete Algorithms, 2019.

[11] K. Censor-Hillel, E. Haramaty, and Z. Karnin. Optimal dynamic distributed MIS. In Proc. of the 2016 ACM Symp. on Principles of Distributed Computing, PODC '16, pages 217-226, 2016.

[12] M. Charikar and S. Solomon. Fully Dynamic Almost-Maximal Matching: Breaking the Polynomial Worst-Case Time Barrier. In 45th International Colloquium on Automata, Languages, and Programming (ICALP 2018), pages 33:1-33:14, 2018.

[13] L. Chitnis, A. Das Sarma, A. Machanavajjhala, and V. Rastogi. Finding connected components in map-reduce in logarithmic rounds. In Proc. of the 2013 IEEE International Conference on Data Engineering, ICDE '13, pages 50-61, 2013.

[14] A. Czumaj, J. Łącki, A. Mądry, S. Mitrović, K. Onak, and P. Sankowski. Round compression for parallel matching algorithms. In Proc. of the 50th Annual ACM SIGACT Symp. on Theory of Computing, STOC 2018, pages 471-484, 2018.

[15] C. Demetrescu and G. F. Italiano. A new approach to dynamic all pairs shortest paths. Fournal of the ACM (FACM), 51(6):968-992, 2004.

[16] M. Ghaffari, T. Gouleakis, C. Konrad, S. Mitrović, and R. Rubinfeld. Improved massively parallel computation algorithms for MIS, Matching, and Vertex cover. In Proc. of the 2018 ACM Symp. on Principles of Distributed Computing, PODC '18, pages $129-138,2018$.

[17] M. Ghaffari and J. Uitto. Sparsifying distributed algorithms with ramifications in massively parallel computation and centralized local computation. In Proc. of the 30th Annual ACM-SIAM Symp. on Discrete Algorithms, pages 1636-1653, 2019.

[18] M. T. Goodrich, N. Sitchinava, and Q. Zhang. Sorting, searching, and simulation in the mapreduce framework. In International Symp. on Algorithms and Computation, pages 374-383, 2011.

[19] M. R. Henzinger and V. King. Randomized fully dynamic graph algorithms with polylogarithmic time per operation. Fournal of the ACM (JACM), 46(4):502-516, 1999.

[20] J. Holm, K. De Lichtenberg, and M. Thorup. Poly-logarithmic deterministic fully-dynamic algorithms for connectivity, minimum spanning tree, 2-edge, and biconnectivity. Fournal of the ACM ( FACM), 48(4):723-760, 2001.

[21] J. E. Hopcroft and R. M. Karp. An $O\left(n^{5 / 2}\right)$ algorithm for maximum matchings in bipartite graphs. SIAM fournal on computing, 2(4):225-231, 1973.

[22] A. Israeli and A. Itai. A fast and simple randomized parallel algorithm for maximal matching. Information Processing Letters, 22(2):77 - 80, 1986.

[23] H. Karloff, S. Suri, and S. Vassilvitskii. A model of computation for MapReduce. In Proc. of the 21st annual ACM-SIAM Symp. on Discrete Algorithms, pages 938-948, 2010.

[24] J. Łącki, V. Mirrokni, and M. Włodarczyk. Connected components at scale via local contractions. arXiv preprint arXiv:1807.10727, 2018.

[25] S. Lattanzi, B. Moseley, S. Suri, and S. Vassilvitskii. Filtering: a method for solving graph problems in MapReduce. In Proc. of the 23th annual ACM Symp. on Parallelism in algorithms and architectures, pages 85-94. ACM, 2011.

[26] Z. Lotker, B. Patt-Shamir, and S. Pettie. Improved distributed approximate matching. In Proc. of the 20th annual Symp. on Parallelism in algorithms and architectures, pages 129-136. ACM, 2008.

[27] A. Lulli, E. Carlini, P. Dazzi, C. Lucchese, and L. Ricci. Fast connected components computation in large graphs by vertex pruning. IEEE Transactions on Parallel \& Distributed Systems, (1):1-1, 2017.

[28] D. Nanongkai, T. Saranurak, and C. Wulff-Nilsen. Dynamic minimum spanning forest with subpolynomial worst-case update time. In 2017 IEEE 58th Annual Symp. on Foundations of Computer Science (FOCS), pages 950-961, Oct 2017.

[29] O. Neiman and S. Solomon. Simple deterministic algorithms for fully dynamic maximal matching. ACM Transactions on Algorithms (TALG), 12(1):7, 2016.

[30] S. Solomon. Fully dynamic maximal matching in constant update time. In IEEE 57th Annual Symp. on Foundations of Computer Science, pages 325-334, Oct 2016.

[31] G. Yaroslavtsev and A. Vadapalli. Massively parallel algorithms and hardness for single-linkage clustering under $\ell_{p}$ distances. In Proc. of the 35th International Conference on Machine Learning, volume 80, pages 5600-5609, 10-15 Jul 2018. 